PROCEEDINGS OF THE

AMERICAN MATHEMATICAL SOCIETY

Volume 132, Number 12, Pages 3559-3568

S 0002-9939(04)07620-8

Article electronically published on July 12, 2004

\title{
REMARK ON WELL-POSEDNESS FOR THE FOURTH ORDER NONLINEAR SCHRÖDINGER TYPE EQUATION
}

\author{
JUN-ICHI SEGATA
}

(Communicated by David S. Tartakoff)

\begin{abstract}
We consider the initial value problem for the fourth order nonlinear Schrödinger type equation (4NLS) related to the theory of vortex filament. In this paper we prove the time local well-posedness for (4NLS) in the Sobolev space, which is an improvement of our previous paper.
\end{abstract}

\section{INTRODUCTION}

We consider the initial value problem for the fourth order nonlinear Scrödinger type equation (4NLS) of the form:

$$
\begin{cases}i \partial_{t} u+\partial_{x}^{2} u+\nu \partial_{x}^{4} u=F\left(u, \bar{u}, \partial_{x} u, \partial_{x} \bar{u}, \partial_{x}^{2} u, \partial_{x}^{2} \bar{u}\right), & (t, x) \in \mathbf{R} \times \mathbf{R}, \\ u(0, x)=u_{0}(x), & x \in \mathbf{R},\end{cases}
$$

where $u(x, t): \mathbf{R} \times \mathbf{R} \rightarrow \mathbf{C}$ is an unknown function. The nonlinear term $F$ is given by

$$
\begin{aligned}
F\left(u, \bar{u}, \partial_{x} u, \partial_{x} \bar{u}, \partial_{x}^{2} u, \partial_{x}^{2} \bar{u}\right)= & -\frac{1}{2}|u|^{2} u+\lambda_{1}|u|^{4} u+\lambda_{2}\left(\partial_{x} u\right)^{2} \bar{u}+\lambda_{3}\left|\partial_{x} u\right|^{2} u \\
& +\lambda_{4} u^{2} \partial_{x}^{2} \bar{u}+\lambda_{5}|u|^{2} \partial_{x}^{2} u,
\end{aligned}
$$

where $\nu, \mu$ are real constants satisfying $\lambda_{1}=3 \mu / 4, \lambda_{2}=2 \mu-\nu / 2, \lambda_{3}=4 \mu+\nu, \lambda_{4}=$ $\mu, \lambda_{5}=2 \mu-\nu$.

The equation in (1.1) describes the three-dimensional motion of an isolated vortex filament embedded in an inviscid incompressible fluid filling an infinite region. This equation is proposed by Fukumoto and Moffatt [8] as some detailed model taking account of the effect from the higher order corrections of the Da Rios model (cubic nonlinear Schrödinger equation):

$$
i \partial_{t} u+\partial_{x}^{2} u=-\frac{1}{2}|u|^{2} u .
$$

For the physical background we refer to [7] and [8].

To motivate our problem in this paper, we state briefly our previous result associated with the well-posedness of the initial value problem (1.1). The notion of well-posedness used here includes the existence, uniqueness of a solution and

Received by the editors April 30, 2003.

2000 Mathematics Subject Classification. Primary 35Q55.

Key words and phrases. Fourth order nonlinear Schrödinger type equation, local wellposedness. 
continuous dependence upon the initial data. In [14], we proved the time local well-posedness as the initial value problem (1.1) in the usual Sobolev spaces $H^{s}(\mathbf{R})$ with $s \geq 1 / 2$ by imposing the condition $\lambda_{5}=2 \mu-\nu=0$ on the coefficients. It was not clear whether this restriction has any physical interpretations. In the present paper, we eliminate this restriction and guarantee the time local well-posedness for (1.1) in Sobolev spaces not lacking the term $|u|^{2} \partial_{x} u$, i.e., $\lambda_{5}=2 \mu-\nu \neq 0$. Consequently our result improved to include the non-completely integrable case, which appears in the real model.

To state our main result precisely, we introduce some notation and function spaces. For a function $u(x, t)$, we denote by $\hat{u}=\mathcal{F}_{x} u$ the Fourier transforms in the $x$ variable. We denote by $\widehat{\widehat{u}}(\tau, \xi)=\mathcal{F}_{t} \mathcal{F}_{x} u(\tau, \xi)$ the space-time Fourier transform. The operator $D_{x}$ and $\left\langle D_{x}\right\rangle$ are given by $D_{x}=\mathcal{F}_{x}^{-1}|\xi| \mathcal{F}_{x}$ and $\left\langle D_{x}\right\rangle=\mathcal{F}_{x}^{-1}\langle\xi\rangle \mathcal{F}_{x}$, respectively, where $\langle x\rangle=\left(1+|x|^{2}\right)^{1 / 2}$. We abbreviate $L_{t}^{p}\left(\mathbf{R} ; L_{x}^{q}(\mathbf{R})\right)$ as $L_{t}^{p}\left(L_{x}^{q}\right)$ and $H_{t}^{p}\left(\mathbf{R} ; H_{x}^{q}(\mathbf{R})\right)$ as $H_{t}^{p}\left(H_{x}^{q}\right)$, respectively. Let $\psi(t)$ be a smooth cut-off function to the interval $[-1,1]$, i.e., $\psi \in C_{0}^{\infty}(\mathbf{R}), \psi(t) \equiv 1$ for $|t| \leq 1$, and $\psi(t) \equiv 0$ for $|t| \geq 2$. For $\delta>0$, we set $\psi_{\delta}(t)=\psi(t / \delta)$. $W_{\nu}(t)$ is the unitary group generated by the linear equation of (1.1). For a real number $s$, let $s+$ denotes a fixed constant larger than $s$.

The equation (1.1) is rewritten as the following integral equation:

$$
u(t)=W_{\nu}(t) u_{0}-i \int_{0}^{t} W_{\nu}\left(t-t^{\prime}\right) F\left(u, \bar{u}, \partial_{x} u, \partial_{x} \bar{u}, \partial_{x}^{2} u, \partial_{x}^{2} \bar{u}\right)\left(t^{\prime}\right) d t^{\prime},
$$

for $t \in[-T, T]$. Then our main result is the following:

Theorem 1.1. Let $\nu<0$. If $s>7 / 12, b \in(1 / 2,3 / 4)$, then for $u_{0} \in H^{s}(\mathbf{R})$, there exist $T=T\left(\left\|u_{0}\right\|_{H^{s}}\right)>0$ and a unique solution $u(t)$ of the initial value problem (1.1) satisfying

$$
\begin{gathered}
u \in C\left([-T, T] ; H^{s}(\mathbf{R})\right), \\
\psi_{T} W_{\nu}(-t) u \in H_{t}^{b}\left(\mathbf{R} ; H_{x}^{s}(\mathbf{R})\right), \\
\psi_{T} W_{\nu}(-t) F \in H_{t}^{b-1}\left(\mathbf{R} ; H_{x}^{s}(\mathbf{R})\right) .
\end{gathered}
$$

Moreover, given $T^{\prime} \in(0, T)$, two maps $u_{0} \longmapsto$ u from $H^{s}(\mathbf{R})$ to $C\left(\left[-T^{\prime}, T^{\prime}\right] ; H^{s}(\mathbf{R})\right)$ and $u_{0} \longmapsto \psi_{T^{\prime}} W_{\nu}(-t)$ u from $H^{s}(\mathbf{R})$ to $H_{t}^{b}\left(\mathbf{R} ; H_{x}^{s}(\mathbf{R})\right)$ are Lipschitz continuous, respectively.

Remark. By employing an analogous method in Molinet-Saut-Tzvetkov [13], we showed in [14] that the initial value problem for (1.1) cannot be solved by the Picard iterative succession via the corresponding integral equation in the Sobolev space $H^{s}(\mathbf{R})$ with $s<1 / 2$. Therefore there is a gap between the index $s=7 / 12+$ of the Sobolev spaces in Theorem 1.1 and the $s=1 / 2$ suggested by the counterexample. However, assuming that $\lambda_{5}=2 \mu-\nu=0$, we could solve the local well-posedness of the initial value problem (1.1) in $H^{s}(\mathbf{R})$ with $s \geq 1 / 2([14])$.

When $\mu+\nu / 2=0$, it should be remarked that (1.1) is the completely integrable equation (see [7]) and has infinitely many conserved quantities (see [9]); for example,

$$
\begin{aligned}
& \Phi_{1}(u)=\frac{1}{2} \int_{\mathbf{R}}|u|^{2} d x, \quad \Phi_{2}(u)=-\frac{i}{2} \int_{\mathbf{R}}\left(\partial_{x} u\right) \bar{u} d x, \\
& \Phi_{3}(u)=-\frac{1}{2} \int_{\mathbf{R}}\left(\partial_{x}^{2} u\right) \bar{u} d x-\frac{1}{8} \int_{\mathbf{R}}|u|^{4} d x, \cdots .
\end{aligned}
$$


Therefore, if $\mu+\nu / 2=0$, combining the above properties and the GagliardoNirenberg inequality:

$$
\|u\|_{L^{4}}^{4} \leq C\|u\|_{L^{2}}^{3}\left\|\partial_{x} u\right\|_{L^{2}} \leq \frac{C^{2}}{2}\|u\|_{L^{2}}^{6}+\frac{1}{2}\left\|\partial_{x} u\right\|_{L^{2}}^{2},
$$

we can show an a priori bound of the solution in $H^{1}(\mathbf{R})$ for all $t>0$. Hence we have the global well-posedness of the solution in our theorem.

To prove Theorem 1.1, we use the method of Fourier restriction norm introduced by Bourgain [4] and Kenig-Ponce-Vega [11, 12. We define the Fourier restriction space $X_{s, b}^{\nu}$ with $b, s, \nu \in \mathbf{R}$ associated with the equation (1.1) as follows: We denote $\phi_{\nu}(\xi)=\xi^{2}-\nu \xi^{4}$. Then $\tau+\phi_{\nu}(\xi)$ represents the symbol of the linearized equation of (1.1). Let

$$
\begin{aligned}
X_{s, b}^{\nu} & \equiv\left\{u \in \mathcal{S}^{\prime}\left(\mathbf{R}^{2}\right) ;\|u\|_{X_{s, b}^{\nu}}<\infty\right\}, \\
\|u\|_{X_{s, b}^{\nu}} & \equiv\left\|\left\langle\tau+\phi_{\nu}(\xi)\right\rangle^{b}\langle\xi\rangle^{s} \widehat{\widehat{u}}(\xi, \tau)\right\|_{L_{\xi}^{2}\left(L_{\tau}^{2}\right)} \\
& =\left\|W_{\nu}(-t) u(t)\right\|_{H_{t}^{b}\left(H_{x}^{s}\right)} .
\end{aligned}
$$

Then we introduce a new estimate of the maximal function related to the unitary group of the fourth order Schrödinger equation (see Proposition 2.2 in Section 2 below). This estimate enables us to handle the worst term $|u|^{2} \partial_{x}^{2} u$ in the nonlinear terms, and we can show the crucial trilinear estimate relevant to this term.

In the next section, we list some linear estimates including the estimate for the maximal function. In the last section, we show the crucial nonlinear estimate and prove Theorem 1.1.

\section{LineAR ESTIMATES}

In this section, we give the linear estimates needed for the proof of crucial nonlinear estimates (see Propositions 3.1 and 3.2 below). It is convenient to use the following notation for the proof of Proposition 3.2 below: For $b \in \mathbf{R}$ let us define $F_{b}$ by

$$
\widehat{\widehat{F_{b}}}(\tau, \xi)=\frac{f(\tau, \xi)}{\left\langle\tau \pm \phi_{\nu}(\xi)\right\rangle^{b}}
$$

for $f \in \mathcal{S}\left(\mathbf{R}^{2}\right)$.

Lemma 2.1. Let $b>1 / 2$ and $b^{\prime}>1 / 4$. For any $f \in L_{\tau}^{2}\left(L_{\xi}^{2}\right)$, we have

$$
\begin{aligned}
\left\|D_{x}^{-1 / 4} F_{b}\right\|_{L_{x}^{4}\left(L_{t}^{\infty}\right)} & \leq C\|f\|_{L_{\tau}^{2}\left(L_{\xi}^{2}\right)} & & \text { (Kenig-Ruiz estimate), } \\
\left\|D_{x}^{3 / 2} F_{b}\right\|_{L_{x}^{\infty}\left(L_{t}^{2}\right)} & \leq C\|f\|_{L_{\tau}^{2}\left(L_{\xi}^{2}\right)} & & \text { (Kato type smoothing effect), } \\
\left\|D_{x}^{3 / 4} F_{b^{\prime}}\right\|_{L_{x}^{4}\left(L_{t}^{2}\right)} & \leq C\|f\|_{L_{\tau}^{2}\left(L_{\xi}^{2}\right)} & & \text { (Kato type smoothing effect), }
\end{aligned}
$$

where $F_{b}$ is defined by (2.1).

Proof of Lemma 2.1. The estimates (2.2) and (2.3) are due to Kenig-Ponce-Vega [10]. For the proof of those estimates, see Theorem 2.5 and Theorem 4.1 in [10, respectively. The inequality (2.4) follows from the interpolation between (2.3) and the Plancherel identity $\left\|F_{0}\right\|_{L_{x}^{2}\left(L_{t}^{2}\right)}=\|f\|_{L_{\xi}^{2}\left(L_{\tau}^{2}\right)}$.

The next proposition plays an important role in the proof of our main theorem (see the proof of Proposition 3.2 below). 
Proposition 2.2 (Estimate for the maximal function). Let $\rho>1, T>0$. For any $u_{0} \in L_{x}^{2}(\mathbf{R})$ and $F \in L_{x}^{2}\left(L_{T}^{1}\right)$, we have

$$
\begin{aligned}
& \left\|\left\langle D_{x}\right\rangle^{-\rho} W(t) u_{0}\right\|_{L_{x}^{2}\left(L_{T}^{\infty}\right)} \leq C\left\|u_{0}\right\|_{L_{x}^{2}}, \\
& \left\|\left\langle D_{x}\right\rangle^{-2 \rho} \int_{0}^{t} W_{\nu}\left(t-t^{\prime}\right) F\left(t^{\prime}\right) d t^{\prime}\right\|_{L_{x}^{2}\left(L_{T}^{\infty}\right)} \leq C\|F\|_{L_{x}^{2}\left(L_{T}^{1}\right)},
\end{aligned}
$$

where $C>0$ is a constant depending on $T$ and $\rho$.

Corollary 2.3. Let $\rho>1 / 2, T \in(0,1), b>1 / 2$, and $f \in L_{\tau}^{2}\left(L_{\xi}^{2}\right)$ with

$$
\text { supp } \mathcal{F}_{t}^{-1} \mathcal{F}_{x}^{-1} f \subset(-T, T) .
$$

Then, for any $F_{b}$ defined by (2.1), we have

$$
\left\|\left\langle D_{x}\right\rangle^{-\rho} F_{b}\right\|_{L_{x}^{2}\left(L_{t}^{\infty}\right)} \leq C\|f\|_{L_{\tau}^{2}\left(L_{\xi}^{2}\right)} .
$$

A similar result to Proposition 2.2 for the Schrödinger equation is obtained by Constantin-Saut [6], Sjölin [15] and Vega [16. The estimate (2.5) is proved by applying the duality argument to the estimate (2.6).

For the purpose of the proof for the inequality (2.6), it suffices to show that the integral kernel of $\left\langle D_{x}\right\rangle^{-2 \rho} W_{\nu}\left(t-t^{\prime}\right)$ belongs to $L_{x}^{1}\left(L_{T}^{\infty}\right)$. More precisely , we require the following lemma.

Lemma 2.4. Let $\rho>1$. We define the integral kernel of $\left\langle D_{x}\right\rangle^{-2 \rho} W_{\nu}\left(t-t^{\prime}\right)$ as $K\left(t-t^{\prime}, x-y\right)$. Then for some $\varepsilon>0$, we have

$$
\left|K\left(t-t^{\prime}, x-y\right)\right| \leq C\langle x-y\rangle^{-1-\varepsilon},
$$

where $C>0$ is a constant depending only on $T, \rho$ and independent of $t, t^{\prime} \in[0, T]$.

A simple application of Young's inequality and Lemma 2.4 yield (2.6).

Proof of Lemma 2.4 For simplicity, we only show the case $\nu=-1$. Let $\phi(\xi) \equiv$ $\phi_{-1}(\xi)=\xi^{2}+\xi^{4}$. We note that the integral kernel of $\left\langle D_{x}\right\rangle^{-2 \rho} W(t-s)$ is given by

$$
K(\sigma, z)=\frac{1}{2 \pi} \int_{\mathbf{R}} e^{i z \xi-i \sigma \phi(\xi)}\langle\xi\rangle^{-2 \rho} d \xi,
$$

where $\sigma=t-t^{\prime}$ and $z=x-y$.

By differentiating the phase, we have

$$
\frac{d}{d \xi}(z \xi-\sigma \phi(\xi))=z-\sigma\left(\phi^{\prime}(\xi)\right)=-4 \sigma\left(\xi^{3}+\frac{\xi}{2}-\frac{z}{4 \sigma}\right)=-4 \sigma \alpha^{3}\left(\eta^{3}+\frac{\eta}{2 \alpha^{2}}-1\right),
$$

where we put $\frac{z}{4 \sigma}=\alpha^{3}, \xi=\alpha \eta, \alpha \in \mathbf{R}$, and $\phi^{\prime}=\frac{d \phi}{d \xi}$.

Let $p_{j}(j=0,1,2)$ be the roots of the algebraic equation $\eta^{3}+\frac{1}{2 \alpha^{2}} \eta-1=0$, and let $p_{0}$ be the unique real root. We note that the $p_{j}$ 's are depending on $\alpha$, and it is easy to see that $p_{0} \rightarrow 1, p_{1} \rightarrow e^{\frac{\pi i}{3}}, p_{2} \rightarrow e^{\frac{2 \pi i}{3}}$ as $|\alpha| \rightarrow \infty$. Indeed, we have a more precise estimate by Rouché's Theorem as follows: Let $|\alpha|>2$. Then, we have

$$
\left|p_{j}-e^{\frac{\pi j i}{3}}\right|<\frac{1}{|\alpha|^{2}}, \quad j=0,1,2 .
$$

Remark. Since $\eta^{3}+\frac{1}{2 \alpha^{2}} \eta-1=\left(\eta-p_{0}\right)\left(\eta-p_{1}\right)\left(\eta-p_{2}\right)$, (2.9) yields

$$
C_{1}\left|\eta-p_{0}\right|\langle\eta\rangle^{2} \leq\left|\eta^{3}+\frac{1}{2 \alpha^{2}} \eta-1\right| \leq C_{2}\left|\eta-p_{0}\right|\langle\eta\rangle^{2} \quad \text { for } \eta \in \mathbf{R},|\alpha|>2
$$

where $C_{1}, C_{2}$ are independent of $\eta$ and $\alpha$. We often use the above inequality when we consider the estimate of $K(\sigma, z)$. 
We separate into two cases: $|z|>64 T$ and $|z| \leq 64 T$.

The case $|z| \leq 64 T$. It directly follows from the definition of $K(\sigma, z)$ that

$$
|K(z, \sigma)| \leq \frac{1}{2 \pi} \int_{\mathbf{R}}\langle\xi\rangle^{-2 \rho} d \xi \leq C .
$$

The case $|z|>64 T$. We note that $|\alpha|=\left|\frac{z}{4 \alpha}\right|^{1 / 3}>\left|\frac{64 T}{8 T}\right|^{1 / 3}=2$. By the identity,

$$
\frac{d}{d \xi}\left(\xi-\alpha p_{0}\right) e^{i z \xi-i \sigma \phi(\xi)}=\left\{1-4 \sigma i\left(\xi-\alpha p_{0}\right) \prod_{j=0}^{2}\left(\xi-\alpha p_{j}\right)\right\} e^{i z \xi-i \sigma \phi(\xi)},
$$

and integrating by parts, we have

$$
\begin{aligned}
& K(\sigma, z) \\
& =-\frac{1}{2 \pi} \int_{\mathbf{R}} e^{i z \xi-i \sigma \phi(\xi)}\left(\xi-\alpha p_{0}\right) \frac{d}{d \xi}\left\{\frac{1}{1-4 \sigma i\left(\xi-\alpha p_{0}\right) \prod_{j=0}^{2}\left(\xi-\alpha p_{j}\right)}\langle\xi\rangle^{-2 \rho}\right\} d \xi \\
& =\frac{1}{2 \pi} \int_{\mathbf{R}} e^{i z \xi-i \sigma \phi(\xi)}\left\{\frac{8 \sigma i \prod_{j=0}^{2}\left(\xi-\alpha p_{j}\right)}{\left\{1-4 \sigma i\left(\xi-\alpha p_{0}\right) \prod_{j=0}^{2}\left(\xi-\alpha p_{j}\right)\right\}^{2}}\right. \\
& \left.\quad+\frac{4 \sigma i\left(\xi-\alpha p_{0}\right)^{2}\left(2 \xi-\alpha p_{1}-\alpha p_{2}\right)}{\left\{1-4 \sigma i\left(\xi-\alpha p_{0}\right) \prod_{j=0}^{2}\left(\xi-\alpha p_{j}\right)\right\}^{2}}\right\}\langle\xi\rangle^{-2 \rho} d \xi \\
& \quad-\frac{1}{2 \pi} \int_{\mathbf{R}} e^{i z \xi-i \sigma \phi(\xi)} \frac{d-\alpha p_{0}}{1-4 \sigma i\left(\xi-\alpha p_{0}\right) \prod_{j=0}^{2}\left(\xi-\alpha p_{j}\right)} \frac{d}{d \xi}\langle\xi\rangle^{-2 \rho} d \xi \\
& \equiv M_{1}(\sigma, z)+M_{2}(\sigma, z) .
\end{aligned}
$$

For $M_{1}(\sigma, z)$, we apply the inequality (2.10) and separate the result into two terms:

$$
\begin{aligned}
\left|M_{1}(\sigma, z)\right| \leq & C \int_{\mathbf{R}} \frac{\sigma \alpha^{4}\left(\eta-p_{0}\right)^{2}\left(\eta^{2}+1\right)}{\left\{1+C \sigma \alpha^{4}\left|\left(\eta-p_{0}\right)^{2}\left(\eta^{2}+1\right)\right|\right\}^{2}}\langle\alpha \eta\rangle^{-2 \rho} \alpha d \eta \\
\leq & C \int_{\left|\eta-p_{0}\right|<1 / 4} \frac{\sigma \alpha^{4}\left(\eta-p_{0}\right)^{2}}{\left\{1+C \sigma \alpha^{4}\left(\eta-p_{0}\right)^{2}\right\}^{2}}\langle\alpha \eta\rangle^{-2 \rho} \alpha d \eta \\
& +C \int_{\left|\eta-p_{0}\right|>1 / 4} \frac{\sigma \alpha^{4}\left(\eta^{2}+1\right)^{2}}{\left\{1+C \sigma \alpha^{4}\left(\eta^{2}+1\right)^{2}\right\}^{2}}\langle\alpha \eta\rangle^{-2 \rho} \alpha d \eta \\
\equiv & M_{1,1}(\sigma, z)+M_{1,2}(\sigma, z) .
\end{aligned}
$$

Recalling $\rho>1$ and $4 \sigma \alpha^{3}=z$, the first term in the right-hand side of (2.13) is estimated as follows:

$$
\begin{aligned}
M_{1,1}(\sigma, z) & \leq C\langle\alpha\rangle^{-2 \rho} \alpha \int_{|\eta|<1 / 4} \frac{\sigma \alpha^{4} \eta^{2}}{\left(1+c \sigma \alpha^{4} \eta^{2}\right)^{2}} d \eta \\
& =C \sigma^{-1 / 2}\langle\alpha\rangle^{-2 \rho} \alpha^{-1} \\
& \leq C \sigma^{-1 / 2+(2 \rho+1) / 3}|z|^{(-2 \rho-1) / 3} \\
& \leq C\langle T\rangle^{-1 / 2+(2 \rho+1) / 3}|z|^{-1-\varepsilon}, \quad \text { for } \rho>1 .
\end{aligned}
$$


Similarly for the second term,

$$
\begin{aligned}
& M_{1,2}(\sigma, z) \\
& \leq C \int_{\mathbf{R}} \frac{\sigma \alpha^{4}\left(\eta^{2}+1\right)^{2}}{\left\{1+C \sigma \alpha^{4}\left(\eta^{2}+1\right)^{2}\right\}^{2}}\langle\alpha \eta\rangle^{-2 \rho} \alpha d \eta \\
& \leq C \int_{|\eta|<1} \frac{\sigma \alpha^{4}}{\left(1+C \sigma \alpha^{4}\right)^{2}}\langle\alpha \eta\rangle^{-2 \rho} \alpha d \eta \\
& \quad+C \int_{|\eta|>1} \frac{\sigma \alpha^{4} \eta^{4}}{\left(1+C \sigma \alpha^{4} \eta^{4}\right)^{2}} \frac{\alpha}{(\alpha \eta)^{2 \rho}} d \eta \\
& \leq C \sigma^{-1} \alpha^{-4}+C \sigma^{-1} \alpha^{-3-2 \rho} \\
& \leq C\langle T\rangle^{2 \rho / 3}|z|^{-4 / 3} .
\end{aligned}
$$

Combining (2.13)-(2.15), we obtain

$$
\left|M_{1}(\sigma, z)\right| \leq C|z|^{-1-\varepsilon} .
$$

Next, we estimate $M_{2}(\sigma, z)$. We apply an integration by parts to have

$$
\begin{aligned}
& M_{2}(\sigma, z) \\
& =\frac{1}{2 \pi} \int_{\mathbf{R}} e^{i z \xi-i \sigma \phi(\xi)}\left(\xi-\alpha p_{0}\right) \\
& \times \frac{d}{d \xi}\left\{\frac{\xi-\alpha p_{0}}{\left\{1-4 \sigma i\left(\xi-\alpha p_{0}\right) \prod_{j=0}^{2}\left(\xi-\alpha p_{j}\right)\right\}^{2}} \frac{d}{d \xi}\langle\xi\rangle^{-2 \rho}\right\} d \xi \\
& \leq C \int_{\mathbf{R}}\left\{\frac{\alpha\left(\eta-p_{0}\right)}{\left\{1+C \sigma \alpha^{4}\left(\eta-p_{0}\right)^{2}\left(\eta^{2}+1\right)\right\}^{2}}\right. \\
& \left.+\frac{\sigma \alpha^{5}\left(\eta-p_{0}\right)^{3}\left(\eta^{2}+1\right)}{\left\{1+C \sigma \alpha^{4}\left(\eta-p_{0}\right)^{2}\left(\eta^{2}+1\right)\right\}^{3}}\right\}\langle\alpha \eta\rangle^{-2 \rho-1} \alpha d \eta \\
& +C \int_{\mathbf{R}} \frac{\alpha^{2}\left(\eta-p_{0}\right)^{2}}{\left\{1+C \sigma \alpha^{4}\left(\eta-p_{0}\right)^{2}\left(\eta^{2}+1\right)\right\}^{2}}\langle\alpha \eta\rangle^{-2 \rho-2} \alpha d \eta \\
& \equiv M_{2,1}(\sigma, z)+M_{2,2}(\sigma, z) \text {. }
\end{aligned}
$$

The evaluations of $M_{2,1}(\sigma, z)$ and $M_{2,2}(\sigma, z)$ are similar to the estimates of $M_{1,1}(\sigma, z)$ and $M_{1,2}(\sigma, z)$, and we proceed by decomposing the integral interval into $\left|\eta-p_{0}\right|<$ $1 / 4$ and $\left|\eta-p_{0}\right|>1 / 4$. Then we have

$$
\begin{aligned}
& \left|M_{2,1}(\sigma, z)\right| \leq C|z|^{-1-2 \rho / 3}, \\
& \left|M_{2,2}(\sigma, z)\right| \leq C|z|^{-2} .
\end{aligned}
$$

Combining (2.17)-(2.19), we obtain

$$
\left|M_{2}(\sigma, z)\right| \leq C|z|^{-1-\varepsilon} .
$$

Combining (2.12), (2.16), (2.20) and (2.11) we have Lemma 2.4. 


\section{Crucial nonlinear estimates}

In this section, we first state the nonlinear estimates obtained in the paper [14].

Proposition 3.1. Let $\nu<0, s>7 / 12, a<-1 / 4$ and $b>1 / 2$. Then for any $u_{j} \in X_{s, b}^{\nu}$ with supp $u_{j} \subset(-T, T), T \in(0,1)$, we have

$$
\begin{aligned}
\left\|u_{1} \bar{u}_{2} u_{3}\right\|_{X_{s, a}^{\nu}} \leq C \prod_{j=1}^{3}\left\|u_{j}\right\|_{X_{s, b}^{\nu}}, \\
\left\|u_{1} \bar{u}_{2} u_{3} \bar{u}_{4} u_{5}\right\|_{X_{s, a}^{\nu}} \leq C \prod_{j=1}^{5}\left\|u_{j}\right\|_{X_{s, b}^{\nu}}, \\
\left\|\partial_{x} u_{1} \bar{u}_{2} \partial_{x} u_{3}\right\|_{X_{s, a}^{\nu}} \leq C \prod_{j=1}^{3}\left\|u_{j}\right\|_{X_{s, b}^{\nu}}, \\
\left\|u_{1} \partial_{x} \bar{u}_{2} \partial_{x} u_{3}\right\|_{X_{s, a}^{\nu}} \leq C \prod_{j=1}^{3}\left\|u_{j}\right\|_{X_{s, b}^{\nu}}, \\
\left\|u_{1} \partial_{x}^{2} \bar{u}_{2} u_{3}\right\|_{X_{s, a}^{\nu}} \leq C \prod_{j=1}^{3}\left\|u_{j}\right\|_{X_{s, b}^{\nu}} .
\end{aligned}
$$

For the proof of Proposition [3.1, see [14]. The next proposition is the crucial estimate in this paper.

Proposition 3.2. Let $\nu<0, s>7 / 12, a<-1 / 4$ and $b>1 / 2$. Then for any $u_{j} \in X_{s, b}^{\nu}$ with supp $u_{j} \subset(-T, T), T \in(0,1)$, we have

$$
\left\|u_{1} \bar{u}_{2} \partial_{x}^{2} u_{3}\right\|_{X_{s, a}} \leq C \prod_{j=1}^{3}\left\|u_{j}\right\|_{X_{s, b}^{\nu}} .
$$

Remark. Concerning the estimates (3.1)-(3.5), we can show still smaller $s$. However we do not need those estimates for $s \leq 7 / 12$ because of the worst term $|u|^{2} \partial_{x}^{2} u$.

Proof of Proposition [3.2. From the definition of $X_{s, b}^{\nu}$ in (1.4) and duality, the inequality (3.6) is reduced to the following estimate: For any $0 \leq f_{4} \in L_{\tau}^{2}\left(L_{\xi}^{2}\right)$,

$$
\begin{aligned}
I & \equiv \int_{\Gamma_{\tau}} \int_{\Gamma_{\xi}} \frac{\left\langle\xi_{4}\right\rangle^{s}\left|\xi_{3}\right|^{2}}{\left\langle\xi_{1}\right\rangle^{s}\left\langle\xi_{2}\right\rangle^{s}\left\langle\xi_{3}\right\rangle^{s}} \frac{\prod_{j=1}^{4} f_{j}\left(\tau_{j}, \xi_{j}\right)}{\prod_{j=1}^{3}\left\langle\tau_{j}+(-1)^{j} \phi_{\nu}\left(\xi_{j}\right)\right\rangle^{b}\left\langle\tau_{4}+\phi_{\nu}\left(\xi_{4}\right)\right\rangle^{|a|}} \\
& \leq \prod_{j=1}^{4}\left\|f_{j}\right\|_{L_{\tau}^{2}\left(L_{\xi}^{2}\right)} .
\end{aligned}
$$

Here we set

$$
f_{j}(\tau, \xi)=\langle\xi\rangle^{s}\left\langle\tau+(-1)^{j} \phi_{\nu}(\xi)\right\rangle^{b}\left|\hat{\hat{u}}\left((-1)^{j} \tau,(-1)^{j} \xi\right)\right| \quad \text { for } j=1,2,3,
$$

and $\Gamma_{\tau}, \Gamma_{\xi}$ denote the hyperplanes on $\mathbf{R}^{4}$ :

$$
\begin{aligned}
& \Gamma_{\tau}=\left\{\left(\tau_{1}, \tau_{2}, \tau_{3}, \tau_{4}\right) \in \mathbf{R}^{4} ; \tau_{1}+\tau_{2}+\tau_{3}+\tau_{4}=0\right\}, \\
& \Gamma_{\xi}=\left\{\left(\xi_{1}, \xi_{2}, \xi_{3}, \xi_{4}\right) \in \mathbf{R}^{4} ; \xi_{1}+\xi_{2}+\xi_{3}+\xi_{4}=0\right\},
\end{aligned}
$$

respectively. We split the domain of integration $I$ into $\left|\xi_{4}\right| \geq 1$ and $\left|\xi_{4}\right| \leq 1$. 
The case $\left|\xi_{4}\right| \geq 1$. We only prove (3.7) for the case $7 / 12<s<3 / 4$. The case $s \geq$ $3 / 4$ is shown in the same manner. It will be convenient to define $\left|\xi_{\text {max }}\right| \geq\left|\xi_{\text {med }}\right| \geq$ $\left|\xi_{\text {min }}\right|$ to be the maximum, median and minimum of $\left|\xi_{1}\right|,\left|\xi_{2}\right|,\left|\xi_{3}\right|$, respectively. Then

$$
\frac{\left|\xi_{3}\right|^{2}\left\langle\xi_{4}\right\rangle^{s}}{\left\langle\xi_{1}\right\rangle^{s}\left\langle\xi_{2}\right\rangle^{s}\left\langle\xi_{3}\right\rangle^{s}} \leq C \frac{\left|\xi_{3}\right|^{2}\left|\xi_{4}\right|^{3 / 4}}{\left\langle\xi_{1}\right\rangle^{s}\left\langle\xi_{2}\right\rangle^{s}\left\langle\xi_{3}\right\rangle^{s}} \leq C \frac{\left|\xi_{\text {max }}\right|^{2}\left|\xi_{4}\right|^{3 / 4}}{\left|\xi_{\text {med }}\right|^{1 / 4}\left\langle\xi_{\text {min }}\right\rangle^{3 s-3 / 4}\left|\xi_{\text {max }}\right|^{1 / 2}} .
$$

Without loss of generality, we may assume $\left|\xi_{1}\right|=\left|\xi_{\text {min }}\right|,\left|\xi_{2}\right|=\left|\xi_{\text {med }}\right|$ and $\left|\xi_{3}\right|=$ $\left|\xi_{\max }\right|$. Let

$$
\hat{\hat{F}}_{j, b}(\tau, \xi)=\frac{f_{j}\left(\tau_{j}, \xi_{j}\right)}{\left\langle\tau_{j}+(-1)^{j} \phi_{\nu}\left(\xi_{j}\right)\right\rangle^{b}}, \quad \text { for } j=1, \cdots, 4 .
$$

Plugging those inequalities (3.8) into $I$ in (3.7) and applying Lemma 2.1 (2.2), (2.3), (2.4), and Corollary 2.3 (2.7), the integral $I$ restricted to this case is bounded by the Hölder inequality so that

$$
\begin{aligned}
& \int_{\Gamma_{\tau}} \int_{\Gamma_{\xi}} \frac{f_{1}\left(\tau_{1}, \xi_{1}\right)}{\left\langle\tau_{1}-\phi_{\nu}\left(\xi_{1}\right)\right\rangle^{b}\left\langle\xi_{1}\right\rangle^{3 s-\frac{3}{4}}} \frac{f_{2}\left(\tau_{2}, \xi_{2}\right)}{\left\langle\tau_{2}+\phi_{\nu}\left(\xi_{2}\right)\right\rangle^{b}\left|\xi_{2}\right|^{\frac{1}{4}}} \frac{\left|\xi_{3}\right|^{\frac{3}{2}} f_{3}\left(\tau_{3}, \xi_{3}\right)}{\left\langle\tau_{3}-\phi_{\nu}\left(\xi_{3}\right)\right\rangle^{b}} \frac{\left|\xi_{4}\right|^{\frac{3}{4}} f_{4}\left(\tau_{4}, \xi_{4}\right)}{\left\langle\tau_{4}+\phi_{\nu}\left(\xi_{4}\right)\right\rangle^{|a|}} \\
\leq & C \int_{\mathbf{R}^{2}}\left|\left\langle D_{x}\right\rangle^{-3 s+\frac{3}{4}} F_{1, b}(t, x)\left\|D_{x}^{-\frac{1}{4}} F_{2, b}(t, x)\right\| D_{x}^{\frac{3}{2}} F_{3, b}(t, x) \| D_{x}^{\frac{3}{4}} F_{4,|a|}(t, x)\right| d t d x \\
\leq & C\left\|\left\langle D_{x}\right\rangle^{-3 s+\frac{3}{4}} F_{1, b}\right\|_{L_{x}^{2}\left(L_{t}^{\infty}\right)}\left\|D_{x}^{-\frac{1}{4}} F_{2, b}\right\|_{L_{x}^{4}\left(L_{t}^{\infty}\right)}\left\|D_{x}^{\frac{3}{2}} F_{3, b}\right\|_{L_{x}^{\infty}\left(L_{t}^{2}\right)}\left\|D_{x}^{\frac{3}{4}} F_{4,|a|}\right\|_{L_{x}^{4}\left(L_{t}^{2}\right)} \\
\leq & C \prod_{j=1}^{4}\left\|f_{j}\right\|_{L_{\tau}^{2}\left(L_{\xi}^{2}\right)} .
\end{aligned}
$$

Here, we used the fact that $-3 s+3 / 4<-1$.

The Case $\left|\xi_{4}\right| \leq 1$. This case is simpler than the case $\left|\xi_{4}\right| \geq 1$. By the same manner as the preceding case, we may assume $\left|\xi_{1}\right| \leq\left|\xi_{2}\right| \leq\left|\xi_{3}\right|$. Then, we easily see that

$$
\frac{\left|\xi_{3}\right|^{2}\left\langle\xi_{4}\right\rangle^{s}}{\left\langle\xi_{1}\right\rangle^{s}\left\langle\xi_{2}\right\rangle^{s}\left\langle\xi_{3}\right\rangle^{s}} \leq C \frac{\left|\xi_{3}\right|^{2}}{\left\langle\xi_{1}\right\rangle^{s}\left\langle\xi_{2}\right\rangle^{s}\left\langle\xi_{3}\right\rangle^{s}} \leq C \frac{\left|\xi_{\text {max }}\right|^{2}}{\left|\xi_{\text {med }}\right|^{1 / 4}\left|\xi_{\text {min }}\right|^{1 / 4}\left|\xi_{\text {max }}\right|^{1 / 2}}
$$

Combining Lemma 2.1 (2.2),$(2.3)$ and (3.11), the integral $I$ in this case again is estimated by

$$
\begin{aligned}
& \int_{\Gamma_{\tau}} \int_{\Gamma_{\xi}} \frac{f_{1}\left(\tau_{1}, \xi_{1}\right)}{\left\langle\tau_{1}-\phi_{\nu}\left(\xi_{1}\right)\right\rangle^{b}\left|\xi_{1}\right|^{1 / 4}} \frac{f_{2}\left(\tau_{2}, \xi_{2}\right)}{\left\langle\tau_{2}+\phi_{\nu}\left(\xi_{2}\right)\right\rangle^{b}\left|\xi_{2}\right|^{1 / 4}} \frac{\left|\xi_{3}\right|^{3 / 2} f_{3}\left(\tau_{3}, \xi_{3}\right)}{\left\langle\tau_{3}-\phi_{\nu}\left(\xi_{3}\right)\right\rangle^{b}} f_{4}\left(\tau_{4}, \xi_{4}\right) \\
\leq & C \int_{\mathbf{R}^{2}}\left|D_{x}^{-1 / 4} F_{1, b}(t, x)\left\|D_{x}^{-1 / 4} F_{2, b}(t, x)\right\| D_{x}^{3 / 2} F_{3, b}(t, x) \| F_{4,0}(t, x)\right| d t d x \\
\leq & C\left\|D_{x}^{-1 / 4} F_{1, b}\right\|_{L_{x}^{4}\left(L_{t}^{\infty}\right)}\left\|D_{x}^{-1 / 4} F_{2, b}\right\|_{L_{x}^{4}\left(L_{t}^{\infty}\right)}\left\|D_{x}^{3 / 2} F_{3, b}\right\|_{L_{x}^{\infty}\left(L_{t}^{2}\right)}\left\|F_{4,0}\right\|_{L_{x}^{2}\left(L_{t}^{2}\right)} \\
\leq & C \prod_{j=1}^{4}\left\|f_{j}\right\|_{L_{\tau}^{2}\left(L_{\xi}^{2}\right)} .
\end{aligned}
$$

By collecting (3.10) and (3.12), we obtain the desired estimate (3.7). 
Proof of Theorem 1.1. We put $r=\left\|u_{0}\right\|_{H^{s}}$. Now for $T \in(0,1)$, we define

$$
\begin{aligned}
\mathcal{B}(r) & =\left\{u \in \mathcal{S}^{\prime}:\|u\|_{X_{s, b}^{\nu}} \leq 2 C r\right\}, \\
\Phi(u) & =\psi(t) W_{\nu}(t) u_{0}-i \psi(t) \int_{0}^{t} W_{\nu}\left(t-t^{\prime}\right) \psi_{T}\left(t^{\prime}\right) F\left(t^{\prime}\right) d t^{\prime} .
\end{aligned}
$$

By similar arguments as in 4], [11] and [12], we have for $b, b^{\prime}$ with $1 / 2<b<b^{\prime}<3 / 4$ and for $u \in \mathcal{B}(r)$,

$$
\|\Phi(u)\|_{X_{s, b}^{\nu}} \leq C_{0} r+C_{1}\left\|\psi_{T} F\right\|_{X_{s, b}^{\nu}} \leq C_{0} r+C_{1} T^{b^{\prime}-b}\|F\|_{X_{s, b-1}^{\nu}} .
$$

Combining Proposition 3.1 with 3.2 , the right-hand side of $(3.13)$ is bounded by

$$
C_{0} r+C_{1} T^{b^{\prime}-b}\left(\|u\|_{X_{s, b}^{\nu}}^{3}+\|u\|_{X_{s, b}^{\nu}}^{5}\right) \leq C_{0} r+C_{1} T^{b^{\prime}-b}\left(1+r^{2}\right) r^{3} .
$$

Therefore, if we choose $T^{b^{\prime}-b} \leq C_{0}\left\{\left(1+r^{2}\right) r^{2} C_{1}\right\}^{-1}$, then $\Phi(u) \in \mathcal{B}(r)$. Similarly, we can show that $\Phi$ is a contraction on $\mathcal{B}(r)$ by choosing $T>0$ sufficiently small. Therefore Banach's Fixed Point Theorem guarantees the existence of a solution in $\mathcal{B}(r) \subset X_{s, b}^{\nu}$. Concerning the uniqueness of the solution in the whole of $X_{s, b}^{\nu}$, we refer to section 4 in 3 . Similar to $\underline{3}$, we introduce the norm:

$$
\|u\|_{X_{T}}=\inf _{w}\left\{\|w\|_{X_{s, b}^{\nu}}: w \in X_{s, b}^{\nu} \text { such that } u(t)=w(t), t \in[-T, T] \text { in } H^{s}(\mathbf{R})\right\} .
$$

If $\left\|u-u^{\prime}\right\|_{X_{T}}=0$, we have $u(t)=u^{\prime}(t)$ in $H^{s}(\mathbf{R})$ for $t \in[-T, T]$. By similar arguments as in 3 , we reduce the uniqueness. The persistency of a solution follows directly from the Sobolev embedding $H_{t}^{b}\left(\mathbf{R} ; H_{x}^{s}(\mathbf{R})\right) \hookrightarrow C\left(\mathbf{R} ; H_{x}^{s}(\mathbf{R})\right)$.

\section{ACKNowledgments}

I wish to express my sincere gratitude to Professor Takayoshi Ogawa for several discussions and valuable advice. I would also like to thank Professor Yasuhide Fukumoto for letting one know of the paper [9]. I would also like to thank Professor Hideo Takaoka for help and encouragement.

\section{REFERENCES}

[1] Ben-Artzi M., Koch H. and Saut J. C., Dispersion estimates for fourth order Schrödinger equations, C. R. Acad. Sci. Paris Sér. I Math. 330 (2000), 87-92. MR 2001a:35149

[2] Bekiranov D., Ogawa T. and Ponce G., Weak solvability and well posedness of a coupled Schrödinger Korteweg-de Vries equation in the capillary-gravity wave interactions, Proc. Amer. Math. Soc. 125 no.10 (1997), 2907-2919. MR 97m:35238

[3] Bekiranov D., Ogawa T. and Ponce G., Interaction equations for short and long dispersive waves, J. Funct. Anal. 158 (1998), 357-388. MR 99i:35143

[4] Bourgain J., Fourier transform restriction phenomena for certain lattice subsets and applications to nonlinear evolution equations. I Schrödinger equations, II The KdV equation, Geom. Funct. Anal. 3 (1993), 107-156, 209-262. MR 95d:35160a

[5] Colliander J., Keel M., Staffilani G., Takaoka H. and Tao T., A refined global well-posedness result for Schrödinger equations with derivative, SIAM J. Math. Anal. 34 (2002), 64-86. MR 2004c:35381

[6] Constantin P. and Saut J. C., Local smoothing properties of dispersive equations, J. Amer. Math. Soc., 1 (1988), 413-439. MR 89d:35150

[7] Fukumoto Y., Three dimensional motion of a vortex filament and its relation to the localized induction hierarchy, Eur. Phys. J. B. 29 (2002), 167-171.

[8] Fukumoto Y. and Moffatt H. K., Motion and expansion of a viscous vortex ring. Part I. A higher-order asymptotic formula for the velocity, J. Fluid. Mech. 417 (2000), 1-45. MR 2002g:76049 
[9] Langer J. and Perline R., Poisson geometry of the filament equation, J. Nonlinear Sci. 1 (1991), 71-93. MR 92k:58118

[10] Kenig C. E., Ponce G. and Vega L., Oscillatory integrals and regularity of dispersive equations, Indiana Univ. Math. J. 40 (1991), 33-69. MR 92d:35081

[11] Kenig C.E., Ponce G. and Vega L., The Cauchy problem for the Korteweg-de Vries equation in Sobolev spaces of negative indices, Duke Math J. 71 (1993), 1-21. MR 94g:35196

[12] Kenig C. E., Ponce G. and Vega L., A bilinear estimate with applications to the KdV equation, J. Amer. Math. Soc. 9 (1996), 573-603. MR 96k:35159

[13] Molinet L., Saut J. C. and Tzvetkov N., Ill-posedness issues for the Benjamin-Ono and related equations, SIAM J. Math. Anal. 33 (2001), 982-988. MR 2002k:35281

[14] Segata J., Well-posedness for the fourth order nonlinear Schrödinger type equation related to the vortex filament, Diff. Integral Equations 16 (2003), no. 7, 841-864. MR 2004d:35236

[15] Sjölin P., Regularity of solutions to the Schrödinger equation, Duke Math. J. 55 (1987), 699-715. MR 88j:35026

[16] Vega L., Schrödinger equations: pointwise convergence to the initial data, Proc. Amer. Math. Soc. 102 (1988), 874-878. MR 89d:35046

Graduate School of Mathematics, Kyushu University, 10-1, Hakozaki 6-chôme, HigASHI-KU, FUKUOKA 812-8581, JAPAN

E-mail address: segata@math.kyushu-u.ac.jp 